THEORIA ET HISTORIA SCIENTIARUM, VOL. XI

Ed. Nicolaus Copernicus University 2014

First published online 31.01.2015

http://dx.doi.org/10.12775/ths-2014-010

\title{
Agnieszka Dębska
}

Faculty of Psychology

University of Warsaw

debska.agn@gmail.com

\section{Pointing in the right direction. A discussion of The evolution of social communication in primates, eds. M. Pina and N. Gontier}

\begin{abstract}
The Evolution of Social Communication in Primates, edited by Marco Pina and Natalie Gontier is an important contribution to the current debates on language evolution. The volume includes texts discussing the emergence of basic social skills connected with language, arguments for vocal and gestural protolanguage, and theories of development of symbolic and compositional language in the history of humanity. In this review, I present the structure and content of the book, but also highlight issues that reflect key controversies in this research area, such as the transition from gestures to speech and from simple vocalizations to modern language. I discuss one specific issue, pointing in nonhuman primates and human children, to offer some remarks on theoretical and empirical criteria for using psychological concepts in debates on social communication in primates.
\end{abstract}

Keywords: language evolution, evolution of social communication, primates, comparative psychology, pointing.

\section{Introduction}

How did social communication evolve in humans and other primates? To what extent is there evolutionary continuity in the communicative skills of humans and other primates? Assuming that there is continuity, how did 
the simple series of vocalizations and manual gestures present in primates transform into compositional, recursive and symbolic human language? Finally, how can scholars investigate these questions? Which methodologies are available and how do they differ from one another? These questions, and more, are the central focus in the anthology The Evolution of Social Communication in Primates: A Multidisciplinary Approach, edited by Pina and Gontier (2014). The book is an outgrowth and expansion of the 2012 International Conference, From Grooming to Speaking: Recent Trends in Social Primatology and Human Ethology. In contrast to most postconference publications, which merely provide an agglomeration of presented papers, the editors of this anthology thoughtfully composed the structure and content of their book.

In my discussion below, I do not aim to present a comprehensive summary of the collection (a daunting task, given its breadth). Instead, I bring to light a selection of topics that define the key points of the current debate on language evolution, like the transition from gestures to speech and from simple vocalizations to modern language. The specific issue of pointing in nonhuman primates and human children, while not a central focus of the book, is particularly interesting for theoretical reasons: being illustrative of the problems with research on social skills in non-human primates, it serves as an inspiration for my discussion in the second part of this paper.

The Evolution of Social Communication in Primates presents an analysis of language evolution: from the existence of basic social skills, such as joint attention, intentionality, theory of mind, communicative gestures and social learning, through theories of speech evolution which incorporated already existing physical and social ground, to the emergence of full-blown, symbolic and compositional language. The main goal of the editors was to provide an overview of different methodologies and theories on the evolution of language and social communication. The book is divided into four parts.

Part 1 concerns the history of studies on communication in primates, with texts by Blancke (2014) on Lord Monboddo's Ourang-Outnag and by Swart (2014) on the morally ambiguous cross-fostering experiments.

Part 2 considers the elements of social communication, including texts on chimpanzees learning sign language (Jensvold 2014), original theoretical view on the study of communication in primates (Botero 2014), the universality of basic emotions (Gaspar, Esteves and Arriaga 2014), the evolution of joint attention (Racine et al. 2014), and ways of understanding and interpreting mental states in psychology (Nagataki 2014).

Part 3 deliberates the transition from mostly gestural communication to modern language. It contains texts on the bodily mimesis origins of speech 
(Zlatev 2014), and multimodal accounts on language evolution (See 2014; Leavens, Taglialatela and Hopkins 2014).

Part 4 refers to the emergence of modern linguistic structures. We find there texts on evolution of related symbolic mediums, like thinking, language and art (Nolan 2014; Tattersall), the studies of language evolution based on the computational theory (Benítez-Burraco, Mineiro and CastroCaldas 2014) and experimental simulation approach (Tamariz 2014) and the challenges on evolutionary biolinguistics (Boeckx 2014).

\section{Communication: from social to symbolic}

While the book brings together a variety of different perspectives, there are some important ideas that are common to all the authors. Besides understanding language as a medium for abstract thought, most of them understand language as a tool for social communication. They admit that language has to evolve in a social environment and, most interestingly, that modern language is based on the structure of a more primitive form of communication, involving both gestural and verbal modalities. Finally, they mostly agree that the evolution of language is methodologically and theoretically associated with the evolution of abstract thinking and a good theory of language evolution should also refer to the emergence of abstract concepts.

The long-running debate about the nature of proto-language in our hominid ancestors is addressed in the chapters by Zlatev (2014), See (2014) and Leavens et al. (2014), who offer a synthetic solution to the older gestural-versus-vocal origin of language debates by embracing a multimodal approach to the evolution of language. By claiming that communication has, from the very beginning, combined both gestures and vocalizations, they avoid "the greatest unsolved problem for gestural protolanguage theories", as Fitch (2010: 448) puts it: the problem of how gestural protolanguage transitioned into vocal language.

The multi-modal approach endorsed by the authors enables scholars to solve this problem, because there never was a "mute" gestural phase. Communication has always combined manual gestures and vocalizations, and human language today still makes use of both modalities. A relevant evolutionary question that needs to be raised therefore, is not how did manual gestures transition into vocal speech, but how did vocal language become the predominant channel of human language?

The authors point out several reasons for this transition. According to Zlatev (2014: 176), speech is a more economic modality to use in bigger 
groups (for counterarguments see Fitch 2010: 445) and speech is considered to be more symbolic and arbitrary, therefore better suited to "differentiate more clearly between an extensive set of concepts, even when their referents are visually similar." To explain how speech co-evolved with manual signals and eventually became the predominant modality, Leavens et al. (2014) interestingly merge Dunbar's from grooming to gossip approach (1996) and Corballis's from hand to mouth approach (2002). According to Corballis, the brains of hominids were prepared for speech production through the long period of controlling intentional manual gestures by the left hemisphere (grooming is usually performed by the right hand). Dunbar (1996) has developed a theory where grooming, which functions as a tool for social bonding, was gradually replaced with vocalizations when group size increased up to the point when it became too time-consuming to socially bond with all group members individually through grooming. To reinforce the connection between producing vocal and manual intentional signals, the authors argue that the attention-getting calls used by apes to share attention with others are an amplified version of grooming.

Moreover, theories of speech evolution have usually struggled with the assumption that vocalizations are inflexible because they are involuntarily produced. But Leavens and coworkers (2014), as well as See (2014), provide convincing examples that many primates, and especially chimpanzees, have more volitional control over their vocal apparatuses than originally assumed, and they can put them to use to acquire attention from an audience. The debate on whether they are actual examples of intentionality remains open. Leavens says yes, while See, using the criteria developed by Tomasello (2008) to understand gestural signals as intentional, says that the same criteria can be used to understand attention-getting calls as being of an intentional nature. Vocal signals can also be learned and are thus not merely "instinctive". For example, pant hoot calls of wild chimpanzees are group-specific (Crockford et al. 2004). Finally, because there is voluntary control over both intentionally-produced gestures as well as attention-getting calls, there is a factor of choice. Indeed, under experimental conditions, chimpanzees sometimes alternate between vocal and gestural signals to obtain a certain response when it is delayed by experimenters (Leavens et al., 2014).

The explanation of how symbolic and compositional language emerged remains problematic. In the anthology, several authors put forward distinct theories by approaching the problem from within different methodological frameworks. Nolan (2014: 241) states that symbolic art such as cave paintings or symbolic ornaments "preceded, facilitated, and triggered the emergence of language and with it the growth of abstract thought". She based on the fact 
that art as symbolic activity shares much in common with language and thinking. Art, thanks to its semantic-syntactic structures, could be the first stable in time and intersubjective medium for symbolism. Another approach comes from Tamariz (2014), who treats language as a complex adaptive system that evolved via cultural transmission over many generations of speakers. She understands language as a culturally-evolved system that is shaped by its own selective pressures and where different linguistic structures compete to be learned and transmitted among new individuals. This view is interesting because it assumes that language owes its present form to the fact that it is the most convenient form for humans to communicate with each other. Experiments on the pragmatic features of conventional systems (Galantucci 2005; Scott-Phillips et al. 2009) are very informative in extracting the relevant aspects of effective communication. Nonetheless, such research is also risky and tough to interpret, because preferential favoring of linguistic constructs is examined in participants who already have the biological equipment for language and are immersed in modern types of conventional communication. Benítez-Burraco et al. (2014) proposed a different hypothesis. They point out that the uniqueness of modern language is based on its computational features and focus on the evolution of brain structures that are responsible for language processing.

A third major topic in the book concerns an investigation into what the common components of social communication in humans and other primates are. In the field of language origins and evolution as well as social primatology, scholars remain divided on the issue. The selected authors in this anthology mostly agree that we share our ability for social communication with other apes, but they differ in determining demarcation points. Comparative studies (in this book presented by Gaspar et al. (2014) and Jensvold (2014), among others) look for traces of abilities that constitute pre-linguistic social interactions in our nearest cousins. The theories and operationalisation of these skills are mostly borrowed from developmental psychology. For example, Baron-Cohen (1995) distinguished four features that enable humans to participate fully in a social interaction: (1) intentionality detector; (2) eye-direction detector; (3) shared-attention mechanism, and (4) theory of mind mechanism. The question emerged whether these features are universal for humans and are unique to the species.

The great apes are most likely able to understand intentional actions (Call, Hare, Carpenter, and Tomasello, 2004). On the other hand, there is no evidence that they understand beliefs or false beliefs (Kaminski, Call, and Tomasello 2008), which would be the base for ascribing them a theory of mind. There is still a debate as to whether apes can follow eye- 
gaze (Okamoto, Tomonaga, Ishii, Kawai, Tanaka, and Matsuzawa 2002) or if they are directed only by head movement and body posture (Tomasello, Hare, B., Lehmann, H., and Call, J. 2007). However, what may be more important, is that they do understand the perception of others; they do know if a conspecific or a human sees something or not (Call and Tomasello 2008). The ability to engage in shared-attention activities is also in dispute.

\section{Pointing: a closer look}

In the second part of the paper, I would like to take a closer look at the case of pointing in chimpanzees because it seems symptomatic of discussions in the fields of comparative psychology and evolutionary psychology. Pointing is considered a very significant developmental capacity, because it is an indicator of joint attention, a necessary element of referential communication. Canonical pointing is claimed to be a uniquely human state where both parties are aware that they mutually share attention toward a common goal. It seems to be an initial and necessary step to successfully refer to objects in the triadic interaction. Developmentally, these kinds of behaviors emerge early, in the first year of life, and it is claimed to be cross-culturally universal (Butterworth 2003). This has made some nativists argue that children are born with the understanding of intersubjectivity (Trevarthen 2011, discussed in Racine et al. 2014). Many socio-pragmatic theories of word-learning use the fact that children can understand communicative intentions of adults before they start to learn meanings of words (Bloom 2000). If there was convincing evidence of apes actually directing and sharing attention with others, they would have perspectivetaking abilities, the basis of communication acts. Overall, gestural theories of language evolution are often based on the assumption that gestures made by modern apes are similar to the practices in hominids, and are prerequisites for learning language (Fitch 2010: 442). These theories have to prove that pointing in apes is used for communication or directing attention and not only ontogenetic ritualization.

What is interesting is that there is disagreement among authors of the book about the ability to understand and produce pointing in apes. It is not surprising because they represent a variety of theoretical and methodological approaches, so they interpret empirical findings differently. For example, for Racine et al. (2014: 133), it is clear that, "great apes use manual gestures, including pointing gestures". Similarly, Leavens et al. (2014: 182) and Botero (2014: 88) claim that chimpanzees in a captive environment are able 
to redirect the attention of others by pointing. On the other hand, Tamariz (2014) convinces us that apes do not point without training and Nagataki (2014: 154) that chimpanzees cannot be involved in joint attentional interactions. In fact, in the field, there is a fierce and complicated debate as to whether chimpanzees, gorillas and macaques are able to truly engage in joint actions.

It is quite undeniable that, in captive environments, apes do make moves similar to pointing when they want food that is unreachable (Hopkins, Russell, McIntyre, and Leavens 2013). It might still be considered ritualized reaching, but apes behave differently adjusting to the attention of a human in the room (Hopkins, Taglialatela, and Leavens 2007), indicating that those moves are audience-oriented. However, theorists such as Tomasello and his group (e.g., Carpenter and Call 2013) claim that pointing in apes is very different from pointing in human children. They refer to the differentiation between imperative pointing, which is strictly instrumental and a goal-oriented activity, mostly to use people as a social tool for providing the chimpanzee with a piece of food, and declarative pointing, which is performed in the desire to show something to somebody, without instrumental cause, just to share attention. While imperative pointing says "I want that! Go there!", declarative pointing means, "Look at that! Something interesting is going to happen soon!"

There is even more disagreement in the case of pointing recognition in chimpanzees. Hare and Tomasello (2004) showed that chimpanzees are very poor at understanding informative pointing in the collaborative context (where conspecifics or humans showed them the place with hidden food) but they are quite good at recognizing goal-directed movements, when a conspecific or a human ineffectively reaches for the object with hidden food in a competitive context. On the other hand, Hopkins et al. (2013) reports that chimpanzees understand intentions behind the pointing when a human requests a chimpanzee for an object from his/her cage.

Skeptics agree that captive apes might point in an imperative way. There is a report that wild chimpanzees also point to the part of the body where they want to be groomed by conspecifics (Pika and Mitani, 2006). However, there is no convincing evidence that apes point declaratively (or proto-declaratively), only to direct another's attention to the object or event. It is claimed that imperative pointing is not an indicator of intersubjectivity because it does not require an understanding of mental states and could be successful without it. Imperative pointing is often viewed as an effect of ritualization (Brinck 2003), even when it can be extended to new cases (as in Hopkins et al. 2013). In contrast, declarative pointing has been 
seen as a triadic relation, which manifests in pure motivation to share attention. In other words, only this elaborate kind of pointing may be an important indicator of joint attention, which enables us to truly engage in mutual exchanges (Carpenter and Call 2013). Moreover, successful social communication, even non-verbal, needed more than just sharing attention, for instance, the existence of common ground between interlocutors, to solve the problem of ambiguous references as well as the epistemic perspectivetaking during interactions (Clark 1996).

Comparative and evolutionary psychologists who try to describe the basis of social communication in primates meet similar problems to developmental psychologists. They have to study very primitive forms of very sophisticated and language-dependent capacities in individuals who practically do not have any language. It raises a methodological problem: which group of behaviors will give us certainty that primates (and newborns) actually understand others' minds? Scientists want to explain the basis of social communication by ascribing a set of abilities that usually manifest themselves in language (such as referential acts and attention-sharing) to individuals without language. This paradox might be a reason why "most authors are not comfortable attributing these mental faculties to non-human animals" (Botero 2014: 91). However, serious problems are theoretical in nature. How can we define minimal prerequisites of understanding intentions, goal-directed behaviors, communicative acts and beliefs? Is it enough to ascribe a theory of mind to an animal that understands the perception and goals of a conspecific, however, does not understand false beliefs? Is it more in sharing attention than coordinating other's focus toward a desired goal? Or does the second-order representation about mutual knowledge have to be involved in that process?

An interesting methodological proposition for researchers was given by Tomasello, Carpenter and Liszkowski (2007). As we cannot rely on the simple observation of a behavior to judge what its nature is in terms of social motivation and mental content, we have to indirectly compare different skills as a complementary group. For example, there is no sense to ascribe to a child or an ape the ability to intentionally share attention towards a mutual object if there is no pure motivation for cooperation or knowledge about a mutual cognitive perspective.

The discussion about pointing shows that there is a need to develop at least minimal empirical criteria for basic concepts in the theories of communication. The first step in formulating these criteria is to compare the most influential theories and methodologies in the field. The Evolution of Social Communication is an important contribution to this discussion. 
However, the explanation concerning psychological and cultural capacities underlying communication is only a beginning for theories of language evolution. They have to be consistent with the fact that nowadays we use a symbolic and partially arbitrary system of signs with a complicated grammar, which is easily learned by children all over the world. What is the bridge between communication based on iconic and intuitively recognizable gestures that are used to direct the attention of others, and the abstract language that is mostly conventionally connected with the world? What were the selective pressures which contributed to the emergence of that abstract system? These questions are still waiting for answers.

\section{References}

Baron-Cohen, S. (1995). Mindblindness: An essay on autism and theory of mind. Cambridge: Cambridge University Press.

Benítez-Burraco, A., Mineiro, A. and Castro-Caldas, A. (2014). The emergence of modern communication in primates: A Computational Approach. In Pina, M. and Gontier, N. (Eds.). The evolution of social communication in primates: A multidisciplinary approach (pp. 28--312). New York: Springer.

Blancke, S. (2014) Lord Monboddo's Ourang-Outang and the Origin and Progress of Language. In Pina, M. \& Gontier, N. (Eds.). The evolution of social communication in primates: A multidisciplinary approach (pp.31--44). New York: Springer.

Bloom, P. (2000). How children learn the meanings of words. Cambridge, MA: MIT Press.

Boeckx, C. (2014) What Can an Extended Synthesis do for Biolinguistics: On the Needsand Benefits of Eco-Evo-Devo Program. In Pina, M. \& Gontier, N. (Eds.). The evolution of social communication in primates: A multidisciplinary approach (pp.313--326). New York: Springer.

Botero, M. (2014). How primate mothers and infants communicate: characterizing interaction in mother-infant studies. In Pina, M. and Gontier, N. (Eds.). The evolution of social communication in primates: A multidisciplinary approach (pp. 8-100). New York: Springer.

Brinck, I. (2003). The pragmatics of imperative and declarative pointing. Cognitive Science Quarterly 3: 429-446.

Butterworth, G. (2003). Pointing is the royal road to language for babies. In S. Kita (Ed.), Pointing: Where language, culture, and cognition meet (pp. 9-33). Mahwah, NJ, USA: Lawrence Erlbaum Associates, Publishers.

Call, J., Hare, B., Carpenter, M., and Tomasello, M. (2004). 'Unwilling' versus 'unable': Chimpanzees' understanding of human intentional action. Developmental Science, 7 (4): 488-498. 
Call, J., and Tomasello, M. (2008). Does the chimpanzee have a theory of mind? Thirty years later. Trends in Cognitive Science, 12: 187-192.

Carpenter, M., and Call, J. (2013). How joint is the joint attention of apes and human infants? In J. Metcalfe and H. S. Terrace (Eds.), Agency and joint attention (pp. 49-61). New York: Oxford University Press.

Clark, H. H. (1996). Using language. Cambridge: Cambridge University Press.

Corballis, M. C. (2002). From hand to mouth: The origins of language. Princeton University Press: Princeton.

Crockford, C., Herbinger, I., Vigilant, L., and Boesch, C. (2004). Wild chimpanzees produce group-specific calls: A case for vocal learning? Ethology, 110: 221243.

Dunbar, R. (1996). Grooming, gossip and the evolution of language. Cambridge: Harvard University Press.

Fitch, W. T. (2010). The Evolution of Language. Cambridge: Cambridge University Press.

Galantucci, B. (2005). An experimental study of the emergence of human communication systems. Cognitive Science 29 (5): 737-767.

Gaspar, A., Esteves, F. and Arriaga, P. (2014). On prototypical facial expressions versus variation in facial behavior: what have we learned on the "visibility" of emotions from measuring facial actions in humans and apes. In Pina, M. and Gontier, N. (Eds.). The evolution of social communication in primates: A multidisciplinary approach (pp. 10-_126). New York: Springer.

Hare, B., and Tomasello, M. (2004). Chimpanzees are more skillful in competitive than in cooperative cognitive tasks. Animal Behaviour, 68 (3): 571-581.

Hopkins, W. D., Russell, J. L., McIntyre, J., and Leavens, D. A. (2013). Are chimpanzees really so poor at understanding imperative pointing? Some new data and an alternative view of canine and ape social cognition. PLoS ONE, 8 (11): e79338.

Hopkins W. D., Taglialatela J. P., and Leavens D. A. (2007). Chimpanzees differentially produce novel vocalizations to capture the attention of a human. Animal Behaviour, 73: 281-286.

Jensvold, M. L. (2014). Experimental conversations: sign language studies with chimpanzees. In Pina, M. and Gontier, N. (Eds.). The evolution of social communication in primates: A multidisciplinary approach (pp.63-82). New York: Springer.

Kaminski, J., Call, J., and Tomasello, M. (2008). Chimpanzees know what others know, but not what they believe. Cognition 109: 224-234.

Leavens, D.A., Taglialatela, J.P. and Hopkins, W.D. (2014). From grasping to grooming to gossip: innovative use of chimpanzee signals in novel environments supports both vocal and gestural theories of language origins. In Pina, M. and Gontier, N. (Eds.). The evolution of social communication in primates: A multidisciplinary approach (pp. 17-194). New York: Springer.

Nagataki, S. (2014). Describing mental states: from brain science to a science of mind reading, In Pina, M. and Gontier, N. (Eds.). The evolution of social 
communication in primates: A multidisciplinary approach (pp. 14--164). New York: Springer.

Nolan, R. (2014). How did humans become behaviorally modern? Revisiting the "Art First" hypothesis. In Pina, M. and Gontier, N. (Eds.). The evolution of social communication in primates: A multidisciplinary approach (pp. 22-248). New York: Springer.

Okamoto, S., Tomonaga, M., Ishii, K., Kawai, N., Tanaka, M., and Matsuzawa, T. (2002). An infant chimpanzee (Pan troglodytes) follows human gaze. Animal Cognition 5: 107-114.

Pina, M. and Gontier, N. (Eds.). (2014). The evolution of social communication in primates: A multidisciplinary approach. New York: Springer.

Pika, S., and Mitani, J. (2006). Referential gestural communication in wild chimpanzees (Pan troglodytes). Curr Biol. 16 (6): 191-2.

Racine, T.P., Wereha, T.J., Vasileva, O., Tafreshi, D. and Thompson, J.J. (2014). The evolution of joint attention: a review and critique. In Pina, M. and Gontier, N. (Eds.). The evolution of social communication in primates: A multidisciplinary approach (pp. 127-146). New York: Springer.

Scott-Phillips, T. C., Kirby S., and Ritchie, G. R. S. (2009). Signalling signalhood and the emergence of communication. Cognition 113 (2): 226-233.

See, A. (2014). Reevaluating chimpanzee vocal signals: toward a multimodal account of the origins of human communication. In Pina, M. and Gontier, N. (Eds.). The evolution of social communication in primates: A multidisciplinary approach (pp. 195-218). New York: Springer.

Swart, S. (2014) Ferality and Morality: The Politics of the "Forbidden Experiment" in the Twentieth Century. In Pina, M. \& Gontier, N. (Eds.). The evolution of social communication in primates: A multidisciplinary approach (pp. 45-62). New York: Springer.

Tamariz, M. (2014). Experiments and simulations can inform evolutionary theories of the cultural evolution of language. In Pina, M. and Gontier, N. (Eds.). The evolution of social communication in primates: A multidisciplinary approach (pp. 24--288). New York: Springer.

Tattersall, I. (2014) Communication and Human Uniqueness. In Pina, M. \& Gontier, N. (Eds.). The evolution of social communication in primates: A multidisciplinary approach (pp.219--228). New York: Springer.

Tomasello M (2008) Origins of human communication. MIT Press, Cambridge.

Tomasello, M., Carpenter, M., and Liszkowski, U. (2007). A new look at infant pointing. Child Development 78: 705-722.

Tomasello, M., Hare, B., Lehmann, H., and Call, J. (2007). Reliance on head versus eyes in the gaze following of great apes and human infants: The cooperative eye hypothesis. Journal of Human Evolution 52: 314-320.

Trevarthen, C. (2011). What is it like to be a person who knows nothing? Defining the active intersubjective mind of a newborn human being. Infant Child Dev. 20: 119-135. 
Zlatev, J. (2014). Bodily mimesis and the transition to speech, In Pina, M. and Gontier, N. (Eds.). The evolution of social communication in primates: A multidisciplinary approach (pp. 165-178). New York: Springer. 